\title{
Effect of transparent wood on polarization degree of light
}

\author{
Elena Vasileva, ${ }^{1 *}$ Adil Baitenov, ${ }^{1}$ HuI Chen ${ }^{2}$, Yuanyuan LI ${ }^{2}$, Ilya \\ Sychugov, ${ }^{1}$ Max YAN, ${ }^{1}$ Lars Berglund ${ }^{2}$, And Sergei Popov ${ }^{1}$ \\ ${ }^{1}$ Department of Applied Physics, KTH - Royal Institute of Technology, Stockholm, Isafjordsgatan 22, 16440, Sweden \\ ${ }^{2}$ Wallenberg Wood Science Center, Department of Fiber and Polymer Technology, KTH - Royal Institute of Technology, Teknikringen 56, 10044, \\ Stockholm, Sweden \\ *Corresponding author: vasileva@kth.se
}

Received XX Month XXXX; revised XX Month, XXXX; accepted XX Month XXXX; posted XX Month XXXX (Doc. ID XXXXX); published XX Month XXXX

\begin{abstract}
We report on the study of polarization properties of light propagating through transparent wood (TW), which an anisotropically scattering medium, and consider two cases: completely polarized and totally unpolarized light. It was demonstrated that scattered light distribution is affected by the polarization state of incident light. Scattering is the most efficient for light polarized parallel to cellulose fibers. Furthermore, unpolarized light becomes partially polarized (with polarization degree of $50 \%$ ) after propagating through the TW. In case of totally polarized incident light, however, the degree of polarization of transmitted light is decreased, for extreme case to a few percent, and reveals an unusual angular dependence on the material orientation. The internal hierarchical complex structure of the material, in particular cellulose fibrils organized in lamellae, is believed to be responsible for the change of light polarization degree. It was demonstrated that the depolarization properties are determined by the angle between the polarization of light and the wood fibers, emphasizing the impact of their internal structure, unique for different wood species.
\end{abstract}

http://dx.doi.org/10.1364/OL.99.099999

Transparent wood (TW) (Fig. 1a) was initially introduced in material science to investigate internal structural features of different wood species [1]. TW is produced from the natural wood via chemical modification including the delignification (removal of the light absorbing lignin from the wood), optional surface modification of cells walls (acetylation), and infiltration of the porous wood template with a polymer of refractive index matching the average refractive index of wood (1.53) [2-6]. The final TW material is essentially a wood-polymer composite, where the polymer (in our case polymethyl methacrylate PMMA) fills void space in the original wood structure.

In recent years, TW has stimulated research interest as an emerging functional optical material $[7,8]$. The material has a unique hierarchical and anisotropic structure (Fig. 1b), which creates the interest in light interaction with this matter. It was demonstrated that transparent wood is a highly scattering material, with high optical transmission, which can reach almost
90\% (for samples of $1 \mathrm{~mm}$ thick balsa TW)[9]. Light scattering is caused by several reasons: refractive index mismatch between the infiltrated polymer and wood components [6], residual air-filled voids after polymer infiltration, and wood-polymer interface gaps formed by polymer shrinkage during polymerization [9].

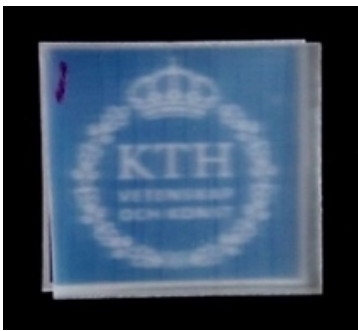

a)

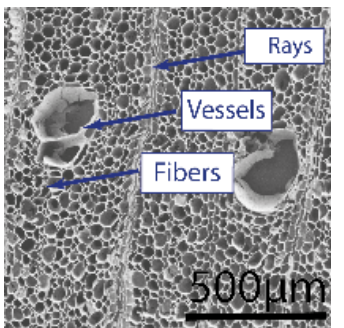

b)
Fig. 1. a) A sample of delignified balsa TW [2] (vessels are vertical) of thickness about $1 \mathrm{~mm}$ placed on top of printed "KTH" logo; b) Cross-sectional image of a delignified template of balsa wood with the main "tubular" features: vessels, fibers (oriented perpendicular to the cross-sectional plane), and rays (located in the plane).

Due to natural structural variations and the semi-ordered anisotropic tubular structure, TW is an intermediate case between two well-studied materials with either randomly dispersed or structurally ordered scattering centres. The structural anisotropy of transparent wood is apparent in its highly anisotropic scattered light distribution [10] presented (Fig. 2a) for 1,2mm thick delignified balsa TW produced as in [2]. The distribution was measured with incident linearly polarized $\left(45^{\circ}\right)$ light of He-Ne laser, with details on experimental measurements provided in [11]. Vessels are large tubular cells (see Fig. 1b) and are apparent as thin vertical stripes in Fig. 1a. The orientation of the vessels was chosen as a reference for material position in the experimental study. The wood fibers are almost parallel to vessels with a misalignment angle of about $6^{\circ}$ for balsa wood [12]. As it is seen in Fig. 2a, light is more strongly scattered in the plane perpendicular to the fibers/vessels orientation. In addition, scattering efficiency depends on the polarization plane of light passing through the TW sample (Fig. 2b). Light is more strongly scattered when the linear polarization is along the vessels rather than when it is perpendicular to them.

In order to investigate the influence of the material structure on polarization of light propagating through the TW, we studied the 
distribution of the states of polarization (SOP) and the degree of polarization (DOP) of scattered light over the sample surface. Unpolarized light from a broad band LED (emission line width of $30 \mathrm{~nm}$ ) with the central wavelength of $528 \mathrm{~nm}$ and a beam diameter of $8 \mathrm{~mm}$ passed through the TW sample, and was collected with a CCD camera. The light SOP at each point of the sample surface was reconstructed via estimation of the Stokes parameters with the spatial resolution $(5 \mu \mathrm{m})$ limited by magnification of the imaging optics and CCD pixel size. Since Stokes parameters are the intensity values, a light source with low coherence (LED) was chosen to exclude measurement errors due to possible formation of interference patterns at the sample surface. In addition, we placed a diffused glass plate in front of the sample to make sure the residual spatial coherence is destroyed. The distribution of SOP over CCD area $(3 \times 3 \mathrm{~mm})$, defined by the angle of rotation (Fig. 3a) and the ellipticity angle of polarization ellipse (Fig. 3b), is uniform over the surface in terms of magnitude fluctuation (colour bar). The wood vessels are observed on the spatial distribution of DOP (Fig. 3c) as the areas with the lowest values indicating low scattering there (incident light is unpolarized).

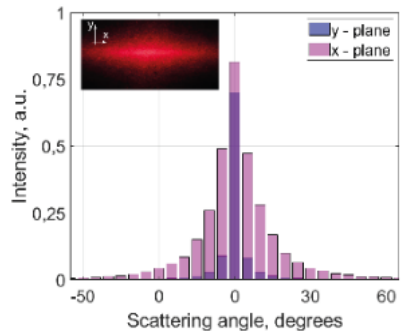

a)

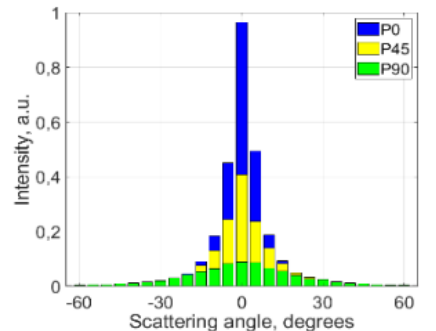

b)

Fig. 2. Normalized angular intensity distribution of scattered light for balsa TW of $1 \mathrm{~mm}$ thickness a) in two orthogonal planes. Input polarization is linear (45 degrees); b) variable linear polarization of incident light: P0 - horizontal, P45 - 45 degrees (pink bar chart on the left figure), P90 - vertical. Vessels are vertically oriented. Light source - He-Ne laser $(633 \mathrm{~nm})$.

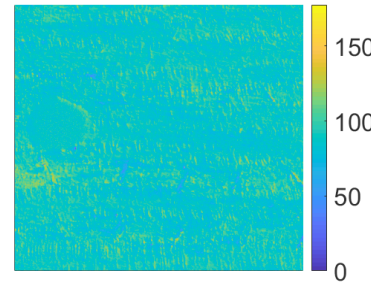

a)

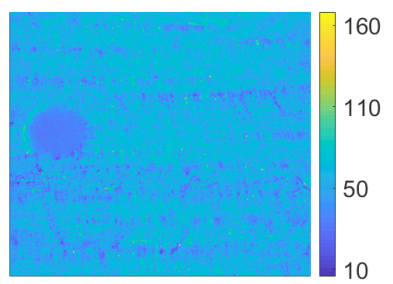

c)

Fig.3. Spatial distribution over the TW surface taken with CCD a) the angle of rotation of polarization ellipse; b) the ellipticity angle of the polarization ellipse; $c$ ) the degree of polarization (DOP). Vessels are horizontally oriented. Light source - green LED (528 nm).
The results obtained by averaging data over the surface of the CCD camera are in good agreement with the measurements obtained with a commercial polarimeter PAX1000VIS from Thorlabs Inc. (Fig. 4). In Fig. 2b, we showed that the part of light with the polarization aligned along vessels and fibers is scattered more efficiently. Thus, unpolarized light, which is a mixture of two orthogonal polarizations, gains some degree of polarization (Fig. 4 a) as it propagates through the TW sample (DOP $\approx 50 \%)$. It is concluded that unpolarized light becomes partially polarized due to anisotropic scattering, where light polarization along the vessels (fibers) is strongly scattered, whereas perpendicular polarization propagates through the sample almost unaffected. With the rotation of the TW sample relatively to the horizontal axis of the lab frame, one can observe the change of the output polarization in the polarized part of scattered light (Fig. 4b). Polarization direction follows the orientation of the vessels (and fibers) of the sample in perpendicular manner.

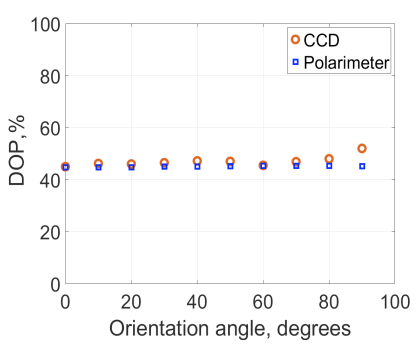

a)

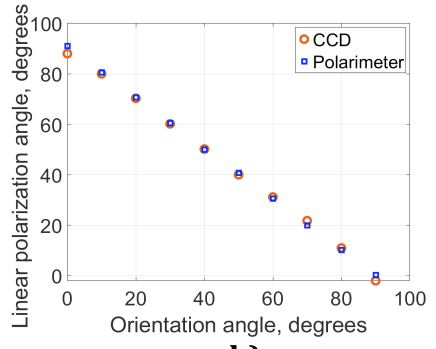

b)
Fig.4. a) Dependence of the DOP (a) and SOP (b) on the orientation of fibers (and vessels) in the TW balsa sample, that is being rotated. Orientation angle is established relatively to the horizontal axis of the lab frame, thus orientation angle of $0^{\circ}$ - corresponds to horizontal orientation of the vessels, and $90^{\circ}$-to vertical. Measurement is done with two techniques - CCD (results are averaged over the CCD area) and the polarimeter. Light source - green LED ( $528 \mathrm{~nm}$ ).

We also investigated the propagation of completely (linearly) polarized light through a sample, in order to better understand the effect of material structure on transmitted light polarization. While unpolarized light becomes partially polarized, the DOP of completely polarized light decreases after transmission through the sample. The depolarization of light depends on light polarization angle with respect to fiber orientation of the wood sample (Fig. 5a). The dependences measured for three different wood species of similar thickness (about $1 \mathrm{~mm}$ ) have two symmetrically located minima at different angles of input polarization $\left(25^{\circ}\right.$ and $\left.155^{\circ}\right)$. Examples of spatial distribution of DOP for two input linear polarizations $\left(25^{\circ}\right.$ and $\left.70^{\circ}\right)$ are presented in Fig. 5b. They differ a lot between each other as well as from the DOP distribution for unpolarized light (Fig.3b). The DOP distribution shows significant decrease (DOP 2-3\%) at the incident light polarization of $25^{\circ}$ for balsa TW, while for the input polarization of $70^{\circ} \mathrm{DOP}$ is mostly $90-100 \%$.

To understand the origin of such an effect, fine structural features of the material should be considered. About $80 \%$ of the TW content is cellulose, located in microfibrils organised in lamellae inside the fiber walls (Fig. 6) [13, 14]. A microfibril has a diameter of around $4 \mathrm{~nm}$. There are three layers of fibrils S1, S2, and S3 in the secondary cell wall, oriented at different angles to the long axis of the large, tubular fiber as they twist helically around the cell (Fig. 6). The layer S2 is the thickest [15]. Fibrils in S2 layer 
are always oriented in right handed helix along the fiber longitudinal direction, while microfibrils in S1 and S3 layers can be oriented in opposite helical directions [15]. The angle of orientation of microfibrils is called microfibril angle (MFA). The MFA in the thickest S2 layer has the strongest effect on physical properties of wood, in particular the axial Young's modulus (stiffness) $[16,17]$. Cellulose (birefringent) crystallites are present in the fibrils. The crystallites consist of highly oriented cellulose molecules with long-range order [18]. Cellulose has permanent electric dipole moment due to asymmetric nature of the polar glucopyranosyl monomers, the chemical polarity of the cellulose chains, and the parallel, non-centrosymmetric structure of the chains in native cellulose inside cellulose crystals [19]. These prolonged large-scale dipoles can efficiently scatter the light of polarization oriented along the dipole axis. The dipoles located in layers S1 - S3 scatter the light most efficiently at angular directions coinciding with the linear polarization direction of the incident light. The contribution of such directional scattering depends on the layer thickness and the density of fibril "helices", and should be taken into account with a proper weighting coefficients. Thus, the DOP dependence on incident polarization (Fig. 5a) is caused by collective light scattering by microfibrils located at different MFAs in the secondary layers (S1-S3). As the MFA values in the layers depend, among other factors, on the wood species and the location of the sample in the tree, the strongest depolarization angles may vary for different types [20] and samples of wood - as, for example, ash, birch, and balsa TW (Fig. 5a) fabricated by the same method as in [2].

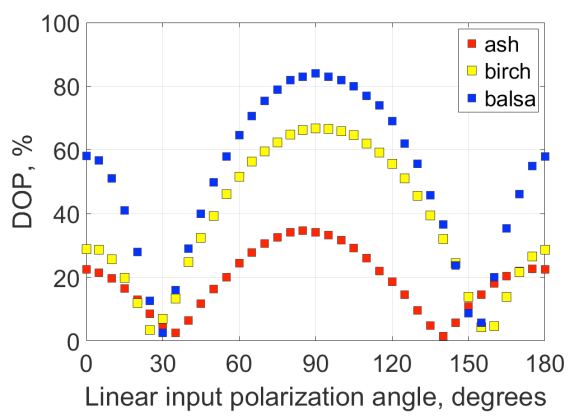

a)

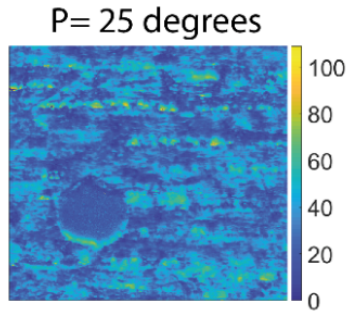

b)

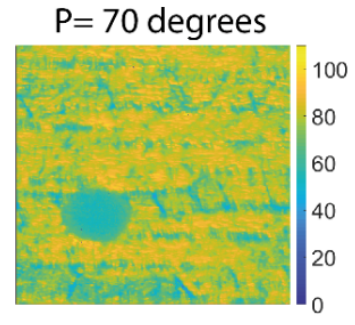

c)
Fig. 5. a) Dependence of DOP from the input linear polarization for different wood types measured with the commercial polarimeter; b) Spatial distribution for DOP in balsa TW over the sample surface for linear input polarization of 25 and c) 70 degrees. The reference mark is seen on the bottom left corner. Vessels are in horizontal orientation. Light source - green LED (528 nm).

To validate the hypothesis of fibril helix angle effect on the DOP of light propagating through the TW, we consider a simplified semi-empirical model. It is based on light scattering by a complex dipole system represented by cellulose crystallites within S-layers, as discussed above. The strength of dipole light scattering is proportional to the projection of the exciting electric field on the dipole axis (Fig. 6). This strength, considering the light intensity, should be proportional to the squared cosine of the angle $\delta$ between the dipole axis and polarization (not shown in Fig. 6 to avoid the overloading with details). The vector of an electric field is in the $x-y$ plane of the lab frame (Fig. 6), and it makes a polarization angle $\gamma$ with $\mathrm{x}$-axis. Incident light ( $\boldsymbol{k}$-vector) is directed along zaxis, and it is always perpendicular to the fibril axis. For simplicity we consider all physical magnitudes in a normalized scale, i.e. equal to unity $|\vec{E}|=|\vec{P}|=1$, and obtain following expression:

$$
\cos (\delta)=\frac{\vec{E} \cdot \vec{P}}{|\vec{E}||\vec{P}|}=E_{x} P_{x}+E_{y} P_{y}+E_{z} P_{z}
$$

There is no projection on z-axis of electric field $E_{z}=0$, whereas

$$
E_{x} P_{x}=|\vec{E}| \cos (\gamma)|\vec{P}| \cos (\alpha)
$$

Since the dipoles follow helical curves in layers S1 - S3, they keep their inclination angle constant within a S-layer relative to the longitudinal direction of the fiber $\alpha$ (i.e. MFA), though continually varying in the laboratory frame, and making angle $\beta$ between zaxis and normal direction to the tangential direction of the dipole (fiber cross-section in Fig. 6; shown for S2 only for simplicity). Thus, at every particular point along the dipole helix

$$
E_{y} P_{y}=|\vec{E}| \sin (\gamma)|\vec{P}| \sin (\alpha) \cos (\beta)
$$

where angle $\beta$ varies from $0^{\circ}$ to $360^{\circ}$ for one full rotation cycle of the helix. Thus, the Eq.1 can be re-written as

$$
\cos (\delta)=\cos (\gamma) \cos (\alpha)+\sin (\gamma) \sin (\alpha) \cos (\beta),
$$

where $\alpha$ is the MFA, $\gamma$ is the polarization angle, $\beta$ - the variable angle relating the direction between a local dipole and field polarization, and $\delta$ - angle between electric field and local dipole (Fig. 6). To calculate the total scattering, the contribution from all dipoles should be integrated along the helix. Since cos is an even function, it is sufficient to integrate over angle $\beta$ in range $0-90^{\circ}$.

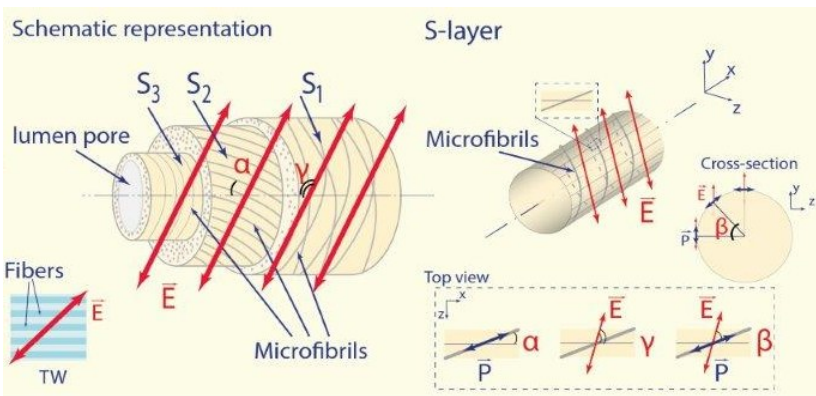

Fig.6. Schematic representation of the cell wall of a tubular wood fiber (lumen pore space in the centre) with three layer of microfibrils (S1 - S3). E - vector of electric field, P - local dipole ;

Equation 4 considers scattering efficiency of an individual Slayer (Fig.7a), as each layer has certain value of MFA. Thus, the total efficiency of dipole excitation across the fiber volume is a sum 
over all three S-layers with the proper weighting coefficients (Fig. 7a). In Fig. 7a, the summarized efficiency of excited dipoles and the total contribution from dipoles (red curve) from three layers S1 S3 are shown for MFAs of $45^{\circ}, 6^{\circ}$ and $80^{\circ}$, respectively, which can occur in balsa [12]. For the balsa wood sample under investigation the MFA angle $\left(6.4^{\circ} \pm 0.4^{\circ}\right)$ of the S2 layer was measured with Wide Angle X-Ray Scattering (WAXS) technique [19, 21].

Since excited dipoles radiate into the space according to a radiation diagram proportional to squared sine from the dipole axis [22], only a small part of the scattered light will reach the detector in a direction perpendicular to the plane of the TW sample. The main part will be scattered into solid angle $4 \pi$. To calculate the DOP of scattered light, the ratio of polarized part $I_{p o l}$ to the total amount of light $I_{\text {tot }}$ reaching the detector should be estimated.

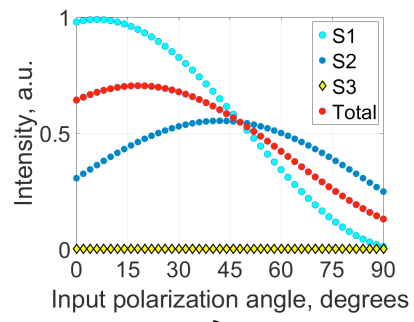

a)

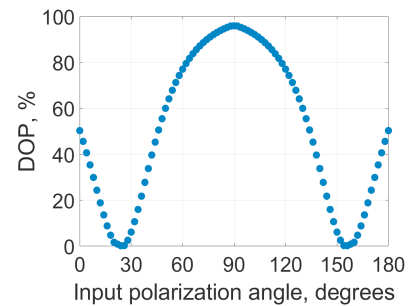

b)
Fig.7. a) Calculated dependence of normalized scattered light intensity from the angle of input linear polarization for each of three secondarylevels of a wood fiber (MFA values $45^{\circ}, 6^{\circ}$ and $80^{\circ}$,) and for the total contribution of all layers; b) Dependence of simulated DOP from the input linear polarization of light.

The intensity of the polarized part can be calculated as the intensity of transmitted light subtracted by the light intensity scattered by dipoles into full solid angle, or, in normalized form $I_{p o l}$. The total intensity collected contains the transmitted light subtracted by the light scattered into the full solid angle, and added with the light scattered according to the dipole radiation diagram into a small solid angle subtended an aperture (diameter of $5 \mathrm{~mm}$ at the distance of $150 \mathrm{~mm}$ ) of the polarimeter

$$
I_{\text {tot }}=I_{p o l}+I_{0} \cdot c=1-I_{d i p} \cdot k+I_{0} \cdot c \text {, }
$$

where $I_{\text {dip }}$ is the intensity of dipole scattering with a coefficient $\mathrm{k}$ depending on scattering cross-section and sample thickness, $I_{0}$ is the intensity of the scattered light reaching the detector area, and $c$ is a coefficient depending on the material thickness for the polarized part of the scattered light. Weighting coefficients for contributions from S1-S2 layers make a basis for a physical background of the model, whereas fitting parameters $c$ and $k$ mainly take into account large scale structure and morphology properties of the material, and are used for the fine adjustment of the model. Figure $7 \mathrm{~b}$ displays a version of the DOP simulated with the properly adjusted coefficients, $k=0,55$ and $c=0,11$. Such simulation gives a reasonable agreement with the experimental data. However, more rigorous modelling of DOP behaviour requires more detailed matching of the material parameters to modelling equations (Eq.1-Eq.6).

In this paper, we report the influence of the transparent wood structure on the properties of light passed through the material for completely polarized and totally unpolarized light. Light scattering properties of TW depend on the polarization state of light. It was found that scattering was most efficient for input light polarization along wood fiber (and vessel) direction. Thus, unpolarized light becomes partially polarized after passing through the material with the DOP increase to $50 \%$. However, for totally polarized incident light (DOP 100\%), the situation is opposite - the degree of polarization of the transmitted light is decreased, for the extreme case to a few percent (DOP 2-3\%). The decrease of DOP depolarization properties of the TW- are determined by the mutual orientation of electric field polarization and direction of the wood fibers, and strong anisotropic scattering of the incident light by cellulose crystallite dipoles making the core structure of the wood material.

Acknowledgements. Authors acknowledges support from The European Research Council, project "Wood NanoTech", gr. \# 742733; Swedish Research Council, project "Electromagnetic coherence: physical effects and applications", gr. \# 621-20124421; The Linné Center in Advanced Optics and Photonics ADOPT at KTH Royal Institute of Technology. Xuan Yang is acknowledged for the help with WAXS measurements.

\section{References}

[1] S. Fink, in: Holzforschung - International Journal of the Biology, Chemistry, Physics and Technology of Wood, 46 (1992) p. 403.

[2] Y. Li, Q. Fu, S. Yu, M. Yan, L. Berglund, Biomacromolecules, 17 (2016), p.1358.

[3] H.S. Yaddanapudi, N. Hickerson, S. Saini, A. Tiwari, Vacuum, 146 (2017), p. 649.

[4] Z. Yu, Y. Yao, J. Yao, L. Zhang, Z. Chen, Y. Gao, H. Luo, J. Mater. Chem. A, 5 (2017), p. 6019.

[5] M. Zhu, T. Li, C.S. Davis, Y. Yao, J. Dai, Y. Wang, F. AlQatari, J.W. Gilman, L. Hu, Nano Energy, 26 (2016), p.332.

[6] Y. Li, E. Vasileva, I. Sychugov, S. Popov, L. Berglund, Adv. Opt. Mat., 6 (2018) 1800059.

[7] Y. Li, S. Yu, J.G.C. Veinot, J. Linnros, L. Berglund, I. Sychugov, Adv. Opt. Mat., 5 (2017) 1600834.

[8] E. Vasileva, Y. Li, I. Sychugov, M. Mensi, L. Berglund, S. Popov, Adv. Opt. Mat., 5 (2017) 1700057.

[9] Y. Li, X. Yang, Q. Fu, R. Rojas, M. Yan, L. Berglund, J. Mater. Chem. A, 6 (2018), p. 1094.

[10] M. Zhu, J. Song, T. Li, A. Gong, Y. Wang, J. Dai, Y. Yao, W. Luo, D. Henderson, L. Hu, Highly Anisotropic, Highly Transparent Wood Composites, Adv. Mat., 28 (2016), p. 5181.

[11] E. Vasileva, H. Chen, Y. Li, I. Sychugov, M. Yan, L. Berglund, S. Popov, Adv. Opt. Mat., 6 (2018) 1800999.

[12] M. Borrega, P. Ahvenainen, R. Serimaa, L.J.W.S. Gibson, Wood Science and Technology, 49 (2015), p. 403.

[13] T. Koddenberg, Handbook of Wood Chemistry and Wood Composites, RC Press, Boca Raton, Florida, (2016).

[14] R.J. Moon, A. Martini, J. Nairn, J. Simonsen, J. Youngblood, Chem. Soc. Rev., 40 (2011), p.3941.

[15] J.R. Barnett, V.A. Bonham, Biological Reviews, 79 (2004) 461-472.

[16] J. Vaněrek, R. Martinek, P. Čada, P. Kuklík, Procedia Engineering, 195 (2017), p. 259.

[17] A. Reiterer, H. Lichtenegger, S. Tschegg, P. Fratzl, Philosophical Magazine A, 79 (1999), p. 2173.

[18] Y. Nishiyama, P. Langan, H. Chanzy, J. Am. Chem. Soc., 124 (2002), p. 9074.

[19] V. Lube, C. Lazarescu, D. Mansfield Shawn, S. Avramidis, in: Holzforschung - Wood Research and Technology, 70 (2016), 485-488. 
[20] H. Lichtenegger, A. Reiterer, S.E. Stanzl-Tschegg, P. Fratzl, J. Struct. Biol., 128 (1999), p. 257.

[21] I.D.J.W.S. Cave, Technology, Theory of X-ray measurement of microfibril angle in wood, 31 (1997), p. 143.

[22] E. Hecht, Optics, 4 ed., Addison-Wesley, New York, 2002.

\section{References}

[1] S. Fink, Transparent Wood - A New Approach in the Functional Study of Wood Structure, Holzforschung - International Journal of the Biology, Chemistry, Physics and Technology of Wood, 46 (1992) 403.

[2] Y. Li, Q. Fu, S. Yu, M. Yan, L. Berglund, Optically Transparent Wood from a Nanoporous Cellulosic Template: Combining Functional and Structural Performance, Biomacromolecules, 17 (2016) 1358-1364.

[3] H.S. Yaddanapudi, N. Hickerson, S. Saini, A. Tiwari, Fabrication and characterization of transparent wood for next generation smart building applications, Vacuum, 146 (2017) 649-654.

[4] Z. Yu, Y. Yao, J. Yao, L. Zhang, Z. Chen, Y. Gao, H. Luo, Transparent wood containing CsxWO3 nanoparticles for heat-shielding window applications, Journal of Materials Chemistry A, 5 (2017) 6019-6024.

[5] M. Zhu, T. Li, C.S. Davis, Y. Yao, J. Dai, Y. Wang, F. AlQatari, J.W. Gilman, L. Hu, Transparent and haze wood composites for highly efficient broadband light management in solar cells, Nano Energy, 26 (2016) 332-339.

[6] Y. Li, E. Vasileva, I. Sychugov, S. Popov, L. Berglund, Optically Transparent Wood: Recent Progress, Opportunities, and Challenges, 6 (2018) 1800059.

[7] Y. Li, S. Yu, J.G.C. Veinot, J. Linnros, L. Berglund, I. Sychugov, Luminescent Transparent Wood, 5 (2017) 1600834.

[8] E. Vasileva, Y. Li, I. Sychugov, M. Mensi, L. Berglund, S. Popov, Lasing from Organic Dye Molecules Embedded in Transparent Wood, 5 (2017) 1700057.

[9] Y. Li, X. Yang, Q. Fu, R. Rojas, M. Yan, L. Berglund, Towards centimeter thick transparent wood through interface manipulation, Journal of Materials Chemistry A, 6 (2018) 1094-1101.

[10] M. Zhu, J. Song, T. Li, A. Gong, Y. Wang, J. Dai, Y. Yao, W. Luo, D. Henderson, L. Hu, Highly Anisotropic, Highly Transparent Wood Composites, 28 (2016) 5181-5187.

[11] E. Vasileva, H. Chen, Y. Li, I. Sychugov, M. Yan, L. Berglund, S. Popov, Light Scattering by Structurally Anisotropic Media: A Benchmark with Transparent Wood, 01800999.

[12] M. Borrega, P. Ahvenainen, R. Serimaa, L.J.W.S. Gibson, Technology, Composition and structure of balsa (Ochroma pyramidale) wood, 49 (2015) 403-420.

[13] T. Koddenberg, Handbook of Wood Chemistry and Wood Composites, RC Press, Boca Raton, Florida, 2016.

[14] R.J. Moon, A. Martini, J. Nairn, J. Simonsen, J. Youngblood, Cellulose nanomaterials review: structure, properties and nanocomposites, Chemical Society Reviews, 40 (2011) 3941-3994.

[15] J.R. Barnett, V.A. Bonham, Cellulose microfibril angle in the cell wall of wood fibres, 79 (2004) 461-472.

[16] J. Vaněrek, R. Martinek, P. Čada, P. Kuklík, The Influence of Microfibril Angle on the Wood Stiffness Parameters, Procedia Engineering, 195 (2017) 259-264.

[17] A. Reiterer, H. Lichtenegger, S. Tschegg, P. Fratzl, Experimental evidence for a mechanical function of the cellulose microfibril angle in wood cell walls, Philosophical Magazine A, 79 (1999) 2173-2184.

[18] Y. Nishiyama, P. Langan, H. Chanzy, Crystal Structure and Hydrogen-Bonding System in Cellulose I $\beta$ from Synchrotron X-ray and Neutron Fiber Diffraction, Journal of the American Chemical Society, 124 (2002) 9074-9082.

[19] V. Lube, C. Lazarescu, D. Mansfield Shawn, S. Avramidis, Wood microfibril angle variation after drying, in: Holzforschung, 2016, pp. 485.
[20] H. Lichtenegger, A. Reiterer, S.E. Stanzl-Tschegg, P. Fratzl, Variation of Cellulose Microfibril Angles in Softwoods and HardwoodsA Possible Strategy of Mechanical Optimization, Journal of Structural Biology, 128 (1999) 257-269.

[21] I.D.J.W.S. Cave, Technology, Theory of X-ray measurement of microfibril angle in wood, 31 (1997) 143-152.

[22] E. Hecht, Optics, 4 ed., Addison-Wesley, New York, 2002. 